\title{
Strong immune function: a benefit promoting the evolution of sociality?
}

\author{
H. Lutermann ${ }^{1} \&$ N. C. Bennett ${ }^{1}$ \\ ${ }^{1}$ Department of Zoology and Entomology, University of Pretoria, Pretoria, South \\ Africa
}

Correspondence

Heike Lutermann, Department of Zoology and Entomology, University of Pretoria, Pretoria 0002, South Africa. Tel:+27 12420 4872; Fax:+27 123625242

Email: hlutermann@zoology.up.ac.za

\section{Abstract}

Life-history theory suggests that breeding effort leads to lowered subsequent fecundity and survival. Immunity is a major physiological mechanism regulating survival and trade-offs between reproductive effort and immune function may be expected. In cooperatively breeding species, breeders may benefit from non-breeders that perform energetically costly tasks by increased survival. However, this has never been related to immune function. In mammals trade-offs between reproductive effort and immune function are likely to be mediated through energy that can be stored in adipose tissue. We compared fat mass and immune function measured as spleen mass between breeders and non-breeders and among the sexes in cooperatively breeding Natal mole-rats Cryptomys hottentotus natalensis. Assuming that larger spleen size indicates a stronger immune function, we hypothesized that reproductive effort leads to reduced energy stores and thus smaller spleen mass in breeders and expected females to have a larger fat and spleen mass than males. We could establish a relationship between energy stores and spleen mass but found the expected sexspecific difference only in breeders. Spleen mass was similar between breeders and non-breeders. The energy cost of reproduction may be compensated for by the contribution of non-breeders in this cooperative breeder. We suggest that nonbreeders reduce work loads for breeders and thereby allow breeders a higher investment in immune function that may result in improved survival. This could also explain increased survival in mole-rats and possibly other cooperative vertebrates.

\section{Introduction}

A major assumption in life-history theory is that increased allocation of resources into one function cannot be achieved without diverting resources from another (Stearns, 1992). A classic example of such a trade-off is the cost of reproduction where breeding effort leads to lowered subsequent fecundity and survival. Immunity is one of the major physiological mechanisms regulating survival and the strength of immune function during breeding may correlate with annual survival (Ardia, Schat \& Winkler, 2003; Hanssen, Folstad \& Erikstad, 2003). 
In most vertebrates, males obtain fitness by increasing the number of matings, whereas females increase their fitness through longevity because their investment in reproduction tends to extend beyond mating (Bateman, 1948; Trivers, 1972). Consequently, females are expected to invest more in immune defence than males to increase their life span (Rolff, 2002). This sex-specific difference in investment in current versus future reproduction may vary according to mating system (polygynous vs. monogamous) and social system (solitary vs. social). In cooperatively breeding species the contribution of non-breeding helpers can reduce the cost of reproduction for breeders (Crick, 1992). However, this possibility has received little attention in cooperative mammals (Solomon \& French, 1997). Russell (2004) suggested that survival benefits of non-breeder contributions are of minor importance in cooperative mammals. This is due to their high fecundity compared with non-cooperative mammals (Russell, 2004) and helpers may rather contribute to productivity (i.e. increased litter sizes). In contrast, cooperatively breeding bird species are characterized by small clutch sizes (Arnold \& Owens, 1998). Moreover, one or both of the breeders may adjust their investment in reproductive effort according to helper contributions and thus increase their survival (Hatchwell, 1999 and references therein). However, this survival benefit has never been related to immune function and little is known about trade-offs between reproductive effort and immune function in cooperative vertebrates.

The most obvious link between reproduction and survival through immune function is energy (Rogowitz, 1996; Niewiarowski, 2001); energy allocated to reproduction is no longer available for self-maintenance, and, as the amount of energy allocated to reproduction increases, so does the magnitude of the costs to survival. On the other hand increases in energy availability by food supplementation can enhance immune function during breeding (Bachman, 2003). Demas, Drazen \& Nelson (2003) showed that surgical removal of fat deposits (i.e. energy reserves) in prairie voles Microtus ochrogaster and Siberian hamsters Phodopus sungorus resulted in lowered humoral immunity. This is mediated by the hormone leptin that is primarily produced by adipose tissue and released in direct proportion to body fat (Demas \& Sakaria, 2005). Hence, body fat appears to be a crucial energy store that mammals rely on to sustain immune function. Thus, larger fat deposits may allow an animal to invest more energy into various components of immune function while breeding.

The aim of our study was to assess for the first time how reproductive effort and the sex of an animal affects energy reserves and an organ of immune system in a cooperatively breeding vertebrate - the Natal mole-rat Cryptomys hottentotus natalensis. Like other social mole-rat species, Natal mole-rats live in family groups and exhibit a reproductive division of labour (Hickman, 1979), allowing measurement of the effects of reproductive effort and sex on immune function by direct comparison among group members.

We assessed immune function in our study species by measuring spleen mass. Spleen size or mass as a measure of immune function has figured prominently in studies on ecology, parasitology and evolution to infer immune system strength (e.g. FernandezLlario et al., 2004; Li et al., 2007; Vicente, Pérez-Rodrígeuz \& Gortazar, 2007). The spleen has an assumed role in lymphocyte recirculation, antibody synthesis and phagocytosis with the consequent destruction of antigens in birds, while in mammals 
it is also involved in red blood cell turnover (John, 1994). The hormone leptin produced by adipose tissue also affects splenic cell-mediated immune function via the central sympathetic nervous system (Okamato et al., 2000) suggesting a functional link between fat deposits and spleen.

It has been suggested by some authors that spleen size correlates with helminth loads (Kristan \& Hammond, 2004; Schwanz, 2006). However, helminth prevalence was low (about 30\%) and we could not find a relationship between helminth infection and spleen mass (H. Lutermann, unpubl. data). Furthermore, postmorten examinations did not suggest any other infections and their subterranean niche exposes them to a limited number of parasites and pathogens; hence, we assumed for our study that a larger spleen mass indicated a higher investment in immune function. This assumption is supported by a recent study by Li et al. (2007) that reports higher levels of serum antibodies against a novel antigen that corresponded with larger spleen masses in Brand's voles Lasiopodomys brandtii. In our study we used measurements of body, fat and spleen mass to explore the following predictions: (1) There is a relationship between energy stores and spleen mass in such that fat mass is positively correlated with spleen mass. (2) As a result of sex-specific reproductive strategies, fat and spleen mass will be reduced in males compared with females. This difference can be expected to be more pronounced in breeders than in non-breeders. (3) If nonbreeders contribute to productivity, higher reproductive effort in larger groups (Russell, 2004) will result in smaller energy stores and lighter spleens of breeders compared with non-breeders. However, if non-breeding helpers 'lighten the load' for breeders as has been proposed by Crick (1992), then breeders and non-breeders should have similar fat and spleen masses.

\section{Materials and methods}

\section{Study site and animals}

Natal mole-rats occur in the in the KwaZulu-Natal and Mpumalanga provinces of South Africa and breed throughout the year (M. K. Oosthuizen, unpubl. data). Information on colony identity, sex, breeding status and body mass as well as preserved alimentary tracts of Natal mole-rats were obtained from animals that had been euthanized for a study of their neuroendocrinology. Animals were trapped on a bimonthly basis from March 2003 to January 2004 on a golf course surrounded by montane grassland at Glengarry Park (1500 $\mathrm{m}$ altitude) in the Kamberg region of KwaZulu-Natal, South Africa $\left(25^{\circ} 58^{\prime} \mathrm{S} ; 2^{\circ} 49^{\prime} \mathrm{E}\right)$. Captures were conducted by exposing mole-rat tunnels close or underneath fresh molehills and setting modified Hickman life-traps (Hickman, 1979) baited with sweet potato at the entrances of those tunnels. All animals were live-trapped. Colony members were housed together in plastic containers until the entire colony had been captured. They were provided with wood shavings as nesting and were fed on sweet potato. A colony was considered to be completely captured if it was functionally complete (i.e. it included a reproductive pair) and if no animals came to the traps for 3 consecutive days after the last individual was caught. Animals were regarded as belonging to the same colony only if they were collected at the same trap site. 


\section{Morphological measurements}

Animals were kept in the laboratory for a maximum of 2 weeks and weighed with a Sartorius 1213MP (Zeiss, Jena, Germany) scale before terminal anaesthesia (halothane). The animals were dissected and the alimentary tract removed and stored in $70 \%$ ethanol. All adipose tissue attached to the alimentary tract was removed and weighed $( \pm 0.01 \mathrm{~g})$ (Ohaus Scout Pro, Pine Brook, NJ, USA). Similarly was the spleen separated from the gut and weighed $( \pm 0.01 \mathrm{~g})$.

\section{Analysis}

Animals were assigned to one of four different groups according to sex and reproductive status. Breeding females (BFs) could readily identified by their perforate vagina and prominent teats while the remaining females were regarded as nonbreeding (NBFs). Following these criteria, a maximum of one BF was identified per colony. Four BF were pregnant and their data were excluded from analyses. One further BF was excluded as her spleen was extremely enlarged for unknown reasons being twice as heavy as the next heaviest of all individuals measured. Male breeders are not as readily identifiable but findings in other mole-rat species suggest that breeding males (BMs) tend to be the largest in a group (Bennett \& Faulkes, 2000). Genetic studies have validated this criterion for other Cryptomys species (Bishop et.al., 2004; Burland et al., 2004) and we therefore used several morphometric criteria to assign males to the breeder category: (1) they had to be among the heaviest individuals in the group; (2) they had to have a minimum body mass of $100 \mathrm{~g}$; however, because some colonies contained several males with very similar body masses we considered a minimum mass difference of $15 \mathrm{~g}$ (corresponding to $>10 \%$ of the average male mass) between the lightest putative BM and the heaviest nonbreeding male (NBM) as an additional criterion to distinguish between potential BM and NBM. Similar to other mole-rat species, this resulted in the identification of one to four males as potential breeders in complete colonies (Bennett \& Faulkes, 2000). Data for body and fat mass were normally distributed while data for spleen mass achieved normality after log transformation. To assess trade-offs between energy reserves and spleen mass we calculated the partial correlation between fat and log spleen mass with body mass as a covariate, thus controlling for confounding effects of this variable. We calculated residuals for fat and log spleen mass from linear regressions of fat and spleen mass, respectively, against body mass. For the comparison of body mass, as well as the residuals of body fat and log spleen mass between reproductive classes and among the sexes, $t$-tests were used. To evaluate the effect of contributions of non-breeders on morphological measures we carried out general linear models (GLMs) separately for body mass, fat mass and log spleen. Only complete colonies were included in these analyses. For all models, sex, breeding status and number of non-breeders were included as independent variables. Additionally, body mass or body and fat mass were included as covariates in GLMs with fat mass and log spleen mass as dependent variables, respectively. Initially we included capture month as an independent variable in all models but eventually discarded it for body mass and log spleen mass, respectively, as it had no significant effect. 


\section{Results}

A total of 283 animals from 59 different colonies were used in this study. Twenty-two colonies were complete and these had an average size of $8.8 \pm 3.7$ adult individuals (range: $2-16$ ). The average number of non-breeders in those colonies was $6.1 \pm 3.4$ (range: 0-12). An additional 22 colonies contained only breeders of one sex and for 15 the breeders of neither sex could be captured. The spleens from 29 animals captured were damaged and these individuals were excluded from analyses involving spleen mass. Independent of body mass there was a significant positive correlation between fat and $\log$ spleen mass $\left(\mathrm{r}_{\mathrm{S}}=0.388\right.$, d.f. $\left.=192, \mathrm{P}<0.0001\right)$ with larger energy stores being associated with heavier spleens (Fig. 1).

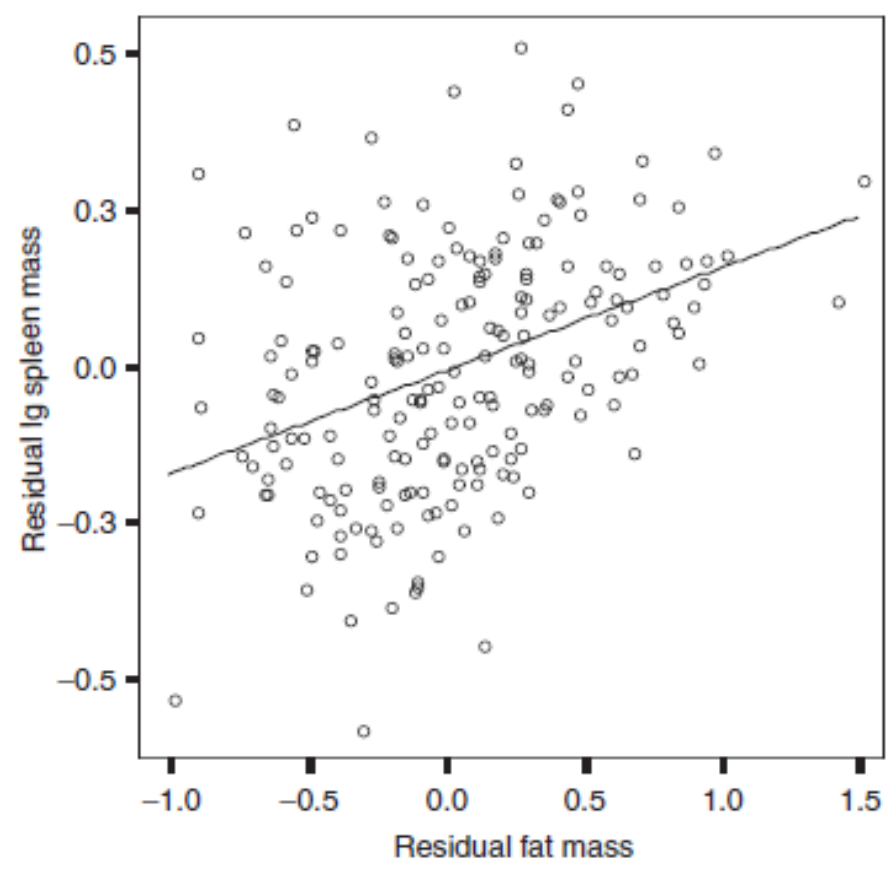

Figure 1 Partial correlation between abdominal fat and log spleen mass $\left(\mathrm{r}_{\mathrm{S}}=0.391\right.$; $\mathrm{P}<0.0001)$.

With an average body mass of $88.4 \pm 21.5 \mathrm{~g}(\mathrm{n}=87)$ NBM were significantly heavier $(\mathrm{t}=-7.87$, d.f. $=190, \mathrm{P}<0.0001$, Fig. $2 \mathrm{a})$ than NBF $(67.8 \pm 14.5, \mathrm{n}=105)$. Absolute fat and spleen mass was $972.9 \mathrm{mg}( \pm 511.9, \mathrm{n}=78)$ and $136.4 \mathrm{mg}( \pm 63.9, \mathrm{n}=70)$, respectively, for NBM compared with $791.9 \mathrm{mg}( \pm 385.5, \mathrm{n}=74)$ and $121.8 \mathrm{mg}( \pm 60.9$, $\mathrm{n}=65$ ) for NBF (Fig. 2b,c). However, there was no difference in their residual fat $(\mathrm{t}=0.18$, d.f. $=149, \mathrm{P}>0.05$, Fig. $3 \mathrm{a})$ or spleen mass $(\mathrm{t}=1.13$, d.f. $=132, \mathrm{P}>0.05$, Fig. $3 \mathrm{~b})$, respectively. Among breeders, males $(125.3 \pm 18.8 \mathrm{~g}, \mathrm{n}=54)$ were significantly heavier $(\mathrm{t}=-6.77$, d.f. $=76, \mathrm{P}<0.0001$, Fig. $2 \mathrm{a})$ than females $(96.0 \pm 14.6 \mathrm{~g}, \mathrm{n}=24)$. Absolute fat and spleen mass was $1105.7 \mathrm{mg}( \pm 575.3, \mathrm{n}=51)$ and $173.1 \mathrm{mg}( \pm 89.0, \mathrm{n}=42)$, respectively for $\mathrm{BM}$ compared with $1007.7 \mathrm{mg}( \pm 615.0, \mathrm{n}=22)$ and $188.4 \mathrm{mg}( \pm 76.0$, $\mathrm{n}=19$ ) for BF (Fig. 2b,c). There was no significant difference in residual fat mass between $\mathrm{BM}$ and $\mathrm{BF}(\mathrm{t}=1.23$, d.f. $=71, \mathrm{P}>0.05$, Fig. $2 \mathrm{~b})$; however, $\mathrm{BF}$ had significantly larger residual spleen masses $(\mathrm{t}=2.62$, d.f. $=59, \mathrm{P}=0.011$, Fig. $2 \mathrm{c})$. 

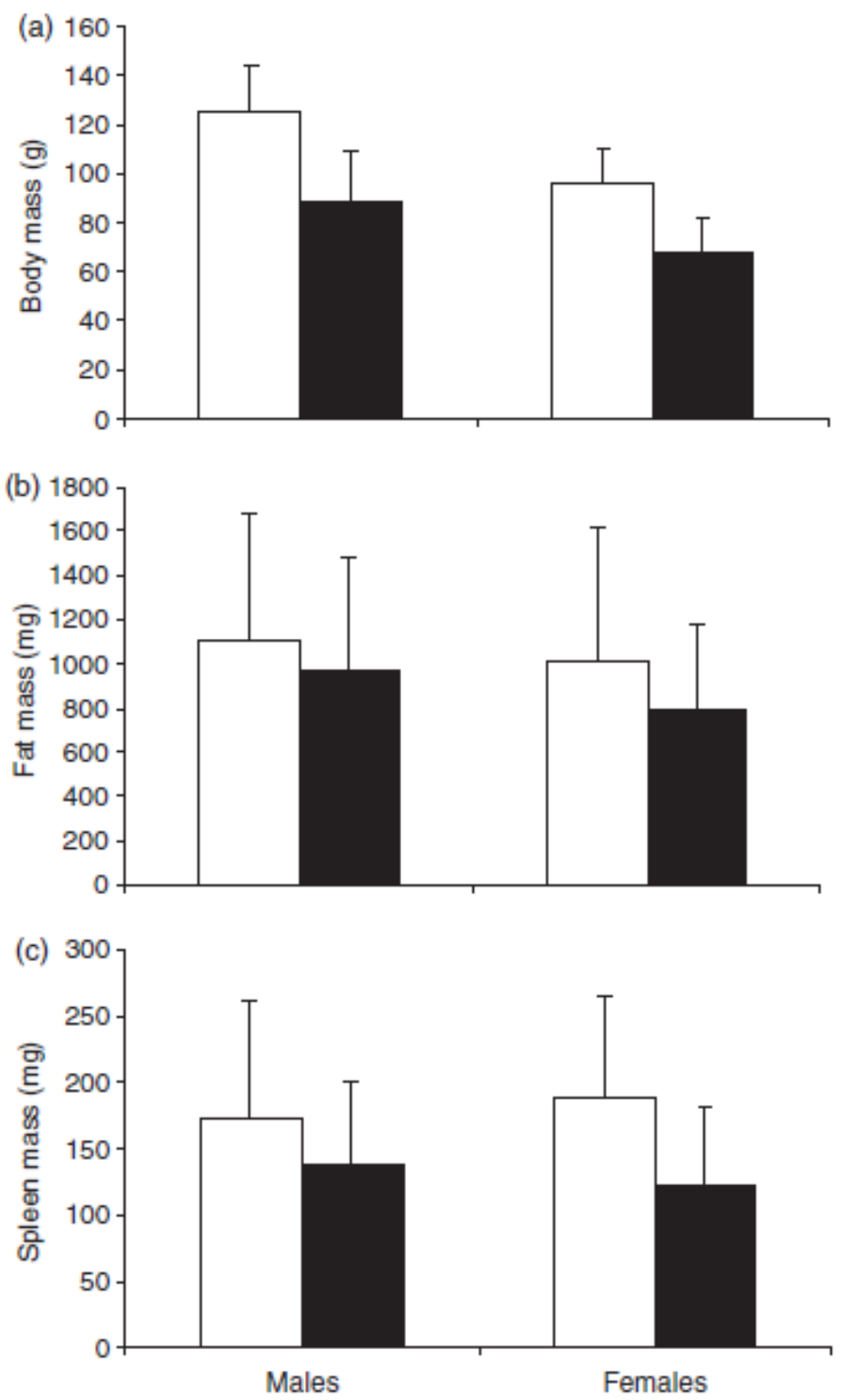

Figure 2 Morphometric data (depicted as mean $\pm \mathrm{sd}$ ) separated according to reproductive status and sex (a) body mass; (b) absolute fat mass; (c) absolute spleen mass. Open bars represent breeders while solid bars indicate non-breeder values. 

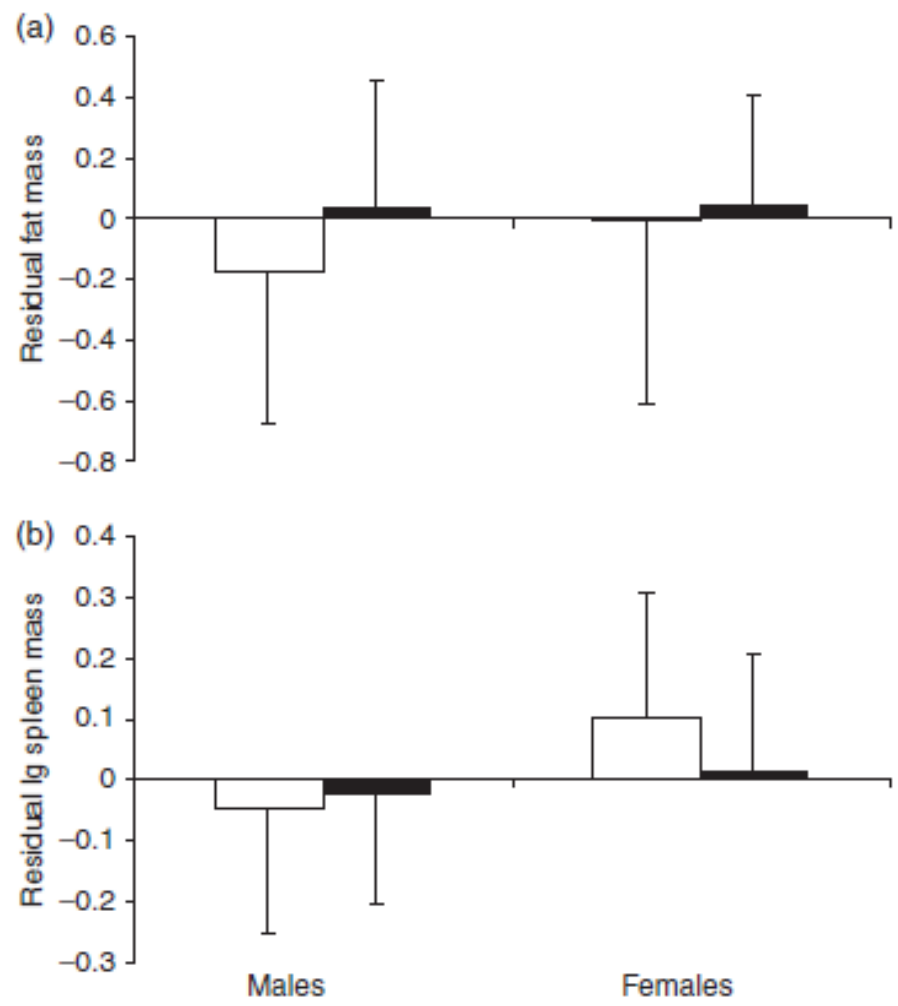

Figure 3 Comparison of body mass independent measures of energy stores and immune function (a) residual fat mass; (b) residual spleen mass (log transformed). Open bars represent breeders while solid bars indicate non-breeder values.

BMs were significantly heavier ( $\mathrm{t}$-test: $\mathrm{t}=-10.37$, d.f. $=139, \mathrm{P}<0.0001$ ) than $\mathrm{NBM}$ as would be expected given our criteria for the assignment of BM. Breeding status did negatively affect energy reserves and NBM had significantly larger residual fat masses than $\mathrm{BM}(\mathrm{t}=2.33$, d.f. $=126, \mathrm{P}=0.021)$. $\mathrm{BM}$ and $\mathrm{NBM}$ did not differ significantly in their residual spleen masses $(t=0.56$, d.f. $=109, \mathrm{P}>0.05)$ and thus breeding status did not appear to affect investment in the immune system in males. Among females, breeders were significantly heavier than non-breeders (t-test: $\mathrm{t}=-8.60$, d.f. $=127, \mathrm{P}<0.0001)$. However, there was no difference in residual fat mass (t-test: $\mathrm{t}=0.38$, d.f. $=94, \mathrm{P}>0.05$ ) between $\mathrm{BF}$ and $\mathrm{NBF}$ and $\mathrm{BF}$ tended to have higher residual spleen masses than NBF, though not significantly so (t-test: $t=-1.71$, d.f. $=82$, $\mathrm{P}=0.091)$.

The GLM (Table 1) confirmed significant differences in body mass according to sex $(\mathrm{F}=46.841$, d.f. $=1, \mathrm{P}<0.0001)$ and reproductive status $(\mathrm{F}=79.056$, d.f. $=1, \mathrm{P}<0.0001)$. In addition, body mass was significantly affected by the number of non-breeders $(\mathrm{F}=4.171$, d.f. $=9, \mathrm{P}<0.0001)$ with a larger number of non-breeders leading to greater body masses. Furthermore the interaction of sex and breeder was significant $(\mathrm{F}=5.579$, d.f. $=1, \mathrm{P}=0.020)$. None of the other interaction terms were significant. When controlling for the effect of body mass, significant effects on energy stores were found for the number of non-breeders $(\mathrm{F}=2.087$, d.f. $=9, \mathrm{P}=0.042)$, capture month 
$(\mathrm{F}=15.556$, d.f. $=5, \mathrm{P}<0.0001)$ and the interaction between sex and reproductive status $(\mathrm{F}=4.774$, d.f. $=1, \mathrm{P}=0.032$, Table 1). During winter months (May-August) fat mass was smaller compared with summer months (November-March). None of the other variables or interaction terms did significantly influence fat mass. A larger body mass resulted in a significantly larger $\log$ spleen mass (Table 1$)(\mathrm{F}=6.676$, d.f. $=1, \mathrm{P}=0.012)$. The covariate body fat significantly influenced the $\log$ spleen mass $(\mathrm{F}=6.218$, d.f. $=1$, $\mathrm{P}=0.015)$. In addition, spleen mass was significantly greater in the presence of nonbreeders $(\mathrm{F}=3.072$, d.f. $=9, \mathrm{P}=0.003)$. The three-way interaction term of sex by breeder by number of helpers nearly reached significance $(\mathrm{F}=2.151$, d.f. $=6, \mathrm{P}=0.056)$. None of the other variables or interaction terms was significant for log spleen mass (Table 1).

Table 1 General linear models of the factors associated with body mass, fat mass and log spleen mass

\begin{tabular}{|c|c|c|c|c|c|c|}
\hline & \multicolumn{2}{|c|}{ Body mass (g) } & \multicolumn{2}{|c|}{ Fat mass $(\mathrm{g})^{\mathrm{a}}$} & \multicolumn{2}{|c|}{$\underline{\text { Log spleen mass }}$} \\
\hline Body mass $(\mathrm{g})^{\mathrm{b}}$ & - & - & 20.440 & $<0.0001^{*}$ & 6.676 & $0.012^{*}$ \\
\hline Sex & 46.841 & $<0.0001^{*}$ & 2.178 & 0.144 & 2.173 & 0.144 \\
\hline Breeder & 79.056 & $<0.0001^{*}$ & 0.031 & 0.861 & 0.021 & 0.843 \\
\hline Number of NB & 4.171 & $<0.0001^{*}$ & 2.087 & $0.042^{*}$ & 3.072 & $0.003^{*}$ \\
\hline Sex $\times$ number of NB & 0.979 & 0.460 & 0.412 & 0.925 & 1.362 & 0.219 \\
\hline Breeder $\times$ number of NB & 0.513 & 0.844 & 0.997 & 0.446 & 1.546 & 0.154 \\
\hline Sex $\times$ breeder $\times$ number of NB & 0.402 & 0.899 & 0.282 & 0.944 & 2.151 & 0.056 \\
\hline
\end{tabular}

* Significant variable.

${ }^{a}$ Results for interactions terms with variable month not displayed.

${ }^{\mathrm{b}}$ Variable included as covariate.

NB, non-breeders.

\section{Discussion}

Trade-offs between energy availability and immune function have been suggested for a number of vertebrates (for a review, see Nelson \& Demas, 1996) and a direct link between fat reserves and the strength of the humoral branch of the immune system in rodents has been demonstrated previously (Demas et al., 2003). Our data suggest a relationship between the mass of adipose tissue and a lymphatic organ - the spleen. Animals with a larger residual fat mass had a larger residual log spleen mass and the fat mass significantly contributed to the variation in log spleen mass in our data. In accordance with these results Vicente et al. (2007) found a correlation between body condition measured as the coefficient of kidney fat mass in relation to kidney mass and spleen mass in red deer Cervus elaphus. These studies are consistent with the hypothesis that mammals exhibit condition-dependent investment into immune function. Although neither study attempted to establish a functional link between fat stores and spleen mass or the strength of the immune system Vicente et al. (2007) also recorded a negative correlation between parasite load and spleen mass. This supports the assumption that larger spleen sizes indicate stronger immune defence. Furthermore, Li et al. (2007) reported a positive relationship between spleen mass and humoral immune function in Brand's voles L. brandtii. Thus, spleen mass could be an 
appropriate measure of immune function in mammals. Although these studies support the use of spleen mass as an indicator of investment in immune function, further research on spleen mass as a measure of immune defence in mammals is needed. Natal mole-rats exhibit a strong sexual dimorphism and as predicted, BM Natal molerats had a lower log spleen masses than BF. This difference between the sexes may be a result of differences in intra-sexual competition. As in other mole-rat species (Bennett \& Faulkes, 2000) there is only one BF per colony while there can be more than one BM. This could lead to competition for matings between BMs with the single BF. The observed sexual mass dimorphism as well as the largest relative testis mass for any mole-rat species (P. W. Bateman, pers. comm.) suggest that intra-sexual competition may well be strong for males possibly leading to increased energy expenditure. Sex-specific differences with regard to energy stores or spleen mass were only apparent in breeders and despite a significant difference in body mass between NBM and NBF they had similar fat and log spleen masses. This contradicts the suggestion that male immune function is compromised in comparison to female immune function as a result of sex-specific reproductive strategies (Rolff, 2002). The lack of sexual dimorphism in the immune measure and energy stores of non-breeders may be a result of social dominance associated with larger body mass (Gabathuler, Bennett \& Jarvis, 1996; Clarke \& Faulkes, 1997, 1998) that may allow them to monopolize food items. Because sex-specific differences are found when comparing $\mathrm{BM}$ and $\mathrm{BF}$ the lack of such sexual dimorphism in non-breeders may simply be a result of the lack of sexual activity. The finding that sexual activity comes at a cost has been shown in a number of species; Klein \& Nelson (1999) reported an impaired immune function only for male meadow voles Microtus pennsylvanicus in mixed-sex pairs but not when kept isolated. Likewise, sex differences in immune function were only apparent while males were sexually active in other species (McKean \& Nunney, 2001; Fernandez-Llario et al., 2004). Thus, our findings suggest sex-specific differences in the costs of reproduction for breeders of the study species.

Despite the assumed costs of reproduction, breeders of both sexes had similar sized (BM) or tended to have larger (BF) spleen masses than their same sex non-breeding counterparts contrasting with reports from other mammal species (Fernandez-Llario et al., 2004; Vicente et al., 2007). At the same time, only BM showed evidence of an associated reduction in energy stores. This suggests only moderate costs of reproduction for $\mathrm{BM}$ and reduced costs in $\mathrm{BF}$. One possible explanation may be that higher competitive ability associated with larger body mass in breeders may enable them to secure more and/or better food items. Hence, breeders may be able to compensate for the energetic costs of reproduction by higher energy intake. Similarly, Bachman (2003) could show that food supplementation did improve immune function in BM Belding's ground squirrels Spermophilus beldingi. In contrast to ground squirrels and the species studied by Fernandez-Llario et al. (2004) and Vicente et al. (2007) mole-rats are not only social but also exhibit a division of labour not found in the other species. Thus, an alternative explanation of the differences in spleen mass found may result from different degrees of engagement in energetically costly activities such as burrow maintenance (Lovegrove, 1989). Such a division of labour has been shown to result in reduced energy expenditure of breeders in Damaraland mole-rats (Scantlebury et al., 2006). Likewise, daily energy expenditure for Natal mole-rat breeders is below that of non-breeders during the dry season $(\mathrm{H}$. Lutermann et al., in prep.). Thus, a larger spleen could be a direct result of 'load-lightening' (Crick, 1992) by non-breeders that allow breeders of the study species to refrain from 
energetically costly activities. Substantial differences in energy expenditure as a result of division of labour have also been shown in another cooperative mammal, the meerkat Suricata suricatta, where breeders largely refrain from the energetically costly babysitting task (Clutton-Brock et al., 1998).

Alternatively, intrinsic differences of individual quality may have led to the observed patterns of a stronger investment in immunity in breeders. Our data represent a snapshot at a single point in the life of the individuals used in this study and we can thus only hypothesize that an increased investment in immune function resulted from attaining a breeding position and recruiting helpers. However, it may generally be the individuals with larger energy stores and stronger immune function that eventually become breeders. Support for this hypothesis comes from another cooperative breeder, the stripe-backed wren Campylorhynchus nuchalis. In this species, helpers with low chances of gaining a breeding position, provision young at high rates and have low survival (Rabenold, 1990) possibly as a result of a trade-off between energy stores and investment in immune function. Load-lightening and differences in intrinsic quality as a cause for the lack of suppression of immune function in breeders are hypotheses that are not mutually exclusive and further study is needed to evaluate them.

If a stronger immune function is associated with increased survival, Natal mole-rat breeders may benefit from the contributions of non-breeders by increased longevity. Long-term survival data for Natal mole-rats are currently unavailable but as in other species of Cryptomys (Bennett \& Faulkes, 2000) litters of Natal mole-rats tend to be small (two to three young, N. C. Bennett, unpubl. data) suggesting that non-breeders do not improve breeder productivity through larger litters. Thus, Russell's (2004) suggestion that non-breeders in cooperative mammals increase productivity rather than survival of breeders may not hold for Natal mole-rats. This conclusion is supported by the remarkable longevity that has been found for social mole-rats (Sherman \& Jarvis, 2002) and in Ansell's mole-rats Cryptomys anselli where breeding activity was associated with a twofold increase in life spans compared with nonbreeders (Dammann \& Burda, 2006). However, currently this hypothesis is speculative and further research is needed to evaluate the hypothesis of a possible link between improved immune function and increased survival in breeders of mole-rats. As with other small mammals (Bartness, Demas \& Song, 2002) fat mass of Natal mole-rats was smaller during winter. Metabolic rates of Natal mole-rats increase with decreasing temperatures (Bennett, Taylor \& Aguilar, 1993) and reductions in fat mass are probably a result of higher thermoregulatory needs during the cold period of the year. The presence of non-breeders can reduce such costs of thermoregulation by the thermoregulatory benefits of huddling that increase with group size (Perret, 1998; Kotze, Bennett \& Scantlebury, in press). The positive effects of non-breeders on body, fat and spleen masses were not restricted to breeders and all colony members benefited from a larger number of non-breeders. Thus, non-breeders may also gain fitness benefits from delaying dispersal.

In conclusion, we were able to establish a relationship between energy stores and spleen mass for Natal mole-rats. Sex-specific differences in spleen mass were only apparent for breeders. Despite the assumed costs of reproduction, no impairment of immune function was found in Natal mole-rat breeders. We propose that this is a result of the contributions of non-breeders that provide energetic benefits for breeders. 
Thus, non-breeders may increase fitness for breeders and this could have played an important role in the evolution of sociality in mole-rats.

\section{References}

- Ardia, D.R., Schat, K.A. \& Winkler, D.W. (2003). Reproductive effort reduces long-term immune function in breeding tree swallows (Tachycineta bicolor). Proc. Roy. Soc. Lond. 270, 1679-1683.

- Arnold, K.E. \& Owens, I.P.F. (1998). Cooperative breeding in birds: a comparative test of the life history hypothesis. Proc. Roy. Soc. Lond. 265, 739-745.

- Bachman, G.C. (2003). Food supplements modulate changes in leucocyte numbers in breeding male ground squirrel. J. Exp. Zool. 206, 2373-2380.

- Bartness, T.J., Demas, G.E. \& Song, C.K. (2002). Seasonal changes in adiposity: the roles of the photoperiod, melatonin and other hormones, and sympathetic nervous system. Exp. Biol. Med. 227, 363-376.

- Bateman, A.J. (1948). Intra-sexual selection in Drosophila. Heredity 2, 349368.

- Bennett, N.C. \& Faulkes, C.G. (2000). African mole-rats - ecology and eusociality. Cambridge: Cambridge University Press.

- Bennett, N.C., Taylor, P.J. \& Aguilar, G.H. (1993). Thermoregulation and metabolic acclimation in the Natal mole-rat (Cryptomys hottentotus natalensis). Z. Säuget. 58, 362-367.

- Bishop, J.M., Jarvis, J.U.M., Spinks, A.C., Bennett, N.C. \& O'Ryan, C. (2004). Molecular insight into patterns of colony composition and paternity in the common mole-rat Cryptomys hottentotus hottentotus. Mol. Ecol. 13, 1217-1229.

- Burland, T.M., Bennett, N.C., Jarvis, J.U.M. \& Faulkes, C.G. (2004). Colony structure and parentage in wild colonies of co-operatively breeding Damaraland mole-rats suggest incest avoidance alone may not maintain reproductive skew. Mol. Ecol. 13, 2371-2379.

- Clarke, F.M. \& Faulkes, C.G. (1997). Dominance and queen succession in captive colonies of the eusocial naked mole-rat, Heterocepalus glaber. Proc. Roy. Soc. Lond. 264, 993-1000.

- Clarke, F.M. \& Faulkes, C.G. (1998). Hormonal and behavioural correlates of male dominance and reproductive status in captive colonies of the naked mole-rat, Heterocephalus glaber. Proc. Roy. Soc. Lond. 265, 1391-1399.

- Clutton-Brock, T.H., Gaynor, D., Kansky, R., MacColl, A.D.C., McIlrath, G.M., Chadwick, P., Brotherton, P.N.M., O'Riain, J.M., Manser, M. \& Skinner, J.D. (1998). Costs of cooperative behaviour in suricates (Suricata suricatta). Proc. Roy. Soc. Lond. 265, 185-190.

- Crick, H.Q.P. (1992). Load-lightening in cooperatively breeding birds and the costs of reproduction. Ibis 134, 56-61.

- Dammann, P. \& Burda, H. (2006). Sexual activity and reproduction delay ageing in a mammal. Curr. Biol. 16, R117-R118.

- Demas, G.E., Drazen, D.L. \& Nelson, R.J. (2003). Reduction in total body fat decrease humoral immunity. Proc. Roy. Soc. Lond. 270, 905-911. 
- Demas, G.E. \& Sakaria, S. (2005). Leptin regulates energetic tradeoffs between body fat and humoural immunity. Proc. Roy. Soc. Lond. 272, 18451850 .

- Fernandez-Llario, P., Parra, A., Cerrato, R. \& de Mendoza, J.H. (2004). Spleen size variations and reproduction in a Mediterranean population of wild boar (Sus scrofa). Eur. J. Wildl. Res. 50, 13-17.

- Gabathuler, U., Bennett, N.C. \& Jarvis, J.U.M. (1996). The social structure and dominance hierarchy of the Mashona mole-rat, Cryptomys darlingi (Rodentia: Bathyergidae) from Zimbabwe. J. Zool. (Lond.) 240, 221-231.

- Hanssen, S.A., Folstad, I. \& Erikstad, K.E. (2003). Reduced immunocompetence and cost of reproduction in common eiders. Oecologia 136, 457-464.

- Hatchwell, B.J. (1999). Investment strategies of breeders in avian cooperative breeding systems. Am. Nat. 154, 205-219.

- Hickman, G.C. (1979). Burrow system structure of the Bathyergid Cryptomys hottentotus in Natal, South Africa. Z. Säuget. 44, 153-162.

- John, J.L. (1994). The avian spleen: a neglected organ. Quart. Rev. Biol. 69, 327-351.

- Klein, S.L. \& Nelson, R.J. (1999). Influence of social factors on immune function and reproduction. Rev. Repr. 4, 168-178.

- Kotze, J., Bennett, N.C. \& Scantlebury, M. (in press) The energetics of huddling in two species of mole-rat (Rodentia: Bathyergidae). Physiol. Behav.

- Kristan, D.M. \& Hammond, K.A. (2004). Morphological plasticity varies with duration of infection: evidence from lactating and virgin wild-derived house mice (Mus musculus) infected with an intestinal parasite (Heligmosomoides polygyrus; Nematoda). J. Exp. Zool. 207, 2351-2360.

- Li, F.-H., Zhong, W.-Q., Wang, Z. \& Wang, D.-H. (2007). Rank in a food competition test and humoral immune functions in male Brandt's voles (Lasiopodomys brandtii). Physiol. Behav. 90, 490-495.

- Lovegrove, B.G. (1989). The cost of burrowing by the social mole-rats (Bathyergidae) Cryptomys damarensis and Heterocephalus glaber: the role of soil moisture. Physiol. Zool. 62, 449-469.

- McKean, K.A. \& Nunney, L. (2001). Increased sexual activity reduces male immune function in Drosophila melanogaster. Proc. Natl. Acad. Sci. USA 98, 7904-7909.

- Nelson, R.J. \& Demas, G.E. 1996. Seasonal changes in immune function. Quart. Rev. Biol. 71, 511-548.

- Niewiarowski, P.H. (2001). Energy budgets, growth rates, and thermal constraints: towards an integrative approach to the study of life-history variation. Am. Nat. 157, 421-433.

- Okamato, S., Irie, Y., Ishikawa, I., Kimura, K. \& Saito, M. (2000). Central leptin suppresses lymphocyte functions through activation of the corticotrophin-releasing hormone-symphathetic nervous system. Brain Res. 855, 192-197.

- Perret, M. (1998). Thermoregulatory advantages for gregarious sleeping in mouse lemurs. J. Mammal. 59, 41-60.

- Rabenold, K.N. (1990). Campylohynus wrens: the ecology of delayed dispersal and cooperation in the Venezuelan savanna. In Cooperative breeding in birds: 159-196. Stacey, P.B. \& Koenig, W.D. (Eds). Cambridge: Cambridge University Press. 
- Rogowitz, G.I. (1996). Trade-offs in energy allocation during lactation. Am. Zool. 36, 197-204.

- Rolff, J. (2002). Bateman's principle and immunity. Proc. Roy. Soc. Lond. 269, 867-872.

- Russell, A.F. (2004). Mammals: comparisons and contrasts. In Ecology and evolution of cooperative breeding in birds: 210-227. Koenig, W.D. \& Dickinson, J.L. (Eds). Berkeley: Cambridge University Press.

- Scantlebury, M., Speakman, J.R., Oosthuizen, M.K., Roper, T.J. \& Bennett, N.C. (2006). Energetics reveals physiologically distinct castes in a eusocial mammal. Nature 440, 795-797.

- Schwanz, L.E. (2006). Schistosome infection in deer mice (Peromyscus maniculatus): impacts on host physiology, behavior and energetics. J. Exp. Biol. 209, 5029-5037.

- Sherman, P.W. \& Jarvis, J.U.M. (2002). Extraordinary life spans of naked mole-rats (Heterocephalus glaber). J. Zool. (Lond.) 46, 307-311.

- Solomon, N.G. \& French, J.A. (1997). Cooperative breeding in mammals. Cambridge: Cambridge University Press.

- Stearns, S.C. (1992). The evolution of life histories. Oxford: Oxford University Press.

- Trivers, R.L. (1972). Parental investment and sexual selection. In Sexual selection and the descent of man: 136-179. Campbell, B. (Ed.). Oxford: Oxford University Press.

- Vicente, J., Pérez-Rodrígeuz, L. \& Gortazar, C. (2007). Sex, age, spleen size, and kidney fat of red deer relative to infection intensities of the lungworm Elaphostrongylus cervi. Naturwissenschaften 94, 581-587. 